\title{
Interleukin-6 and intrapulmonary shunt
}
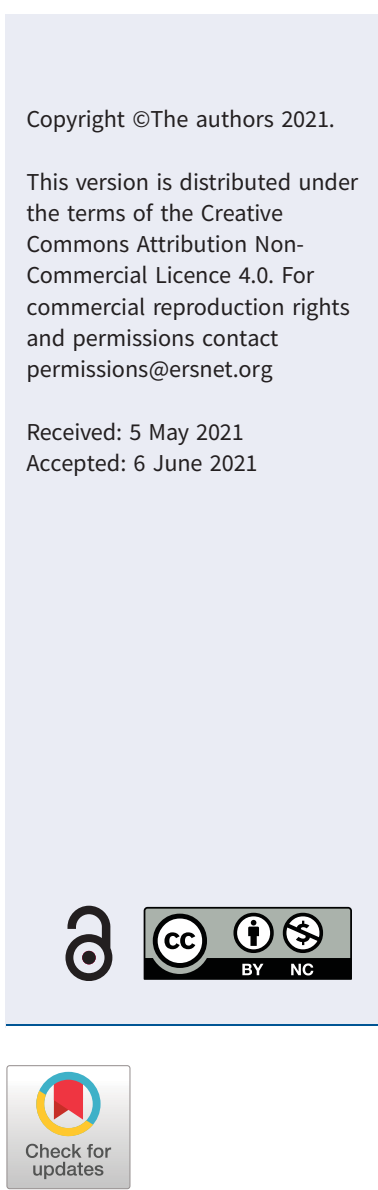

To the Editor:

We read with interest the article by Koтwica et al. [1] showing the utility of clinical pulse oximetry measurements to quantify shunt and ventilation-perfusion mismatch and their predictive value in severe coronavirus disease 2019 (COVID-19). The authors found that shunt correlated with markers of activated inflammatory response (i.e. C-reactive protein) but not those of activated coagulation (such as D-dimer). Their results reinforce the growing evidence for the role of impaired hypoxic pulmonary vasoconstriction (HPV) as a primary cause for severe acute respiratory syndrome coronavirus 2 (SARS-CoV-2)-induced hypoxaemia [2].

We would like to draw the attention of readers to a possible pathophysiological role of interleukin (IL)-6 in intrapulmonary shunt associated with SARS-CoV-2-induced acute respiratory distress syndrome that has been overlooked. IL-6 is a fundamental player in the inflammation associated with COVID-19 and the level of this cytokine serves as a biomarker of poor prognosis [3]. Moreover, although controversy remains regarding the population of patients that may benefit from anti-IL-6 therapies in COVID-19, two large randomised clinical trials have shown reduced mortality in patients treated with tocilizumab $[4,5]$, and seven randomised controlled trials have shown reduced risk of mechanical ventilation [6].

The initial evidence for the involvement of IL-6 in impaired HPV comes from studies in mice and rats. Thus, human recombinant IL-6 inhibited HPV in mice [7]. We also observed that IL-6 inhibited HPV and an antibody against IL-6 prevented the impairment of HPV induced by bacterial endotoxin in isolated rat pulmonary arteries [8]. In addition, several studies have demonstrated a positive impact of tocilizumab on arterial oxygenation in patients with severe COVID-19 $[9,10]$.

In conclusion, we speculate that IL-6 is involved in the impaired HPV associated with COVID-19. The relationship of intrapulmonary shunt with IL-6 levels and with anti-IL-6 therapies deserves further investigation.

Shareable abstract (@ERSpublications)

Based on animal studies and indirect clinical evidence, it may be speculated that IL- 6 has a pathophysiological role in intrapulmonary shunt associated to COVID-19 https://bit.ly/3whQVqd

Cite this article as: Perez-Vizcaino F, Moreno L, Lorente JA. Interleukin-6 and intrapulmonary shunt. Eur Respir J 2021; 58: 2101292 [DOI: 10.1183/13993003.01292-2021].

Francisco Perez-Vizcaino ${ }^{1,2,3}$, Laura Moreno $0^{1,2,3}$ and José A. Lorente $2,4,5$

${ }^{1}$ Dept of Pharmacology and Toxicology, School of Medicine, Universidad Complutense de Madrid, Madrid, Spain. ${ }^{2}$ CIBER de Enfermedades Respiratorias (CIBERES), Madrid, Spain. ${ }^{3}$ Instituto de Investigación Sanitaria Gregorio Marañón (IISGM), Madrid, Spain. ${ }^{4}$ Critical Care Service, Hospital Universitario de Getafe, Madrid, Spain. ${ }^{5}$ Universidad Europea, Madrid, Spain.

Corresponding author: Francisco Perez-Vizcaino (fperez@med.ucm.es)

Conflict of interest: F. Perez-Vizcaino reports fees for lectures from Actelion. L. Moreno has nothing to disclose. J.A. Lorente has nothing to disclose.

\section{References}

1 Kotwica A, Knights $\mathrm{H}$, Mayor $\mathrm{N}$, et al. Intrapulmonary shunt measured by bedside pulse oximetry predicts worse outcomes in severe COVID-19. Eur Respir J 2021; 57: 2003841.

2 Habashi NM, Camporota L, Gatto LA, et al. Functional pathophysiology of SARS-CoV-2-induced acute lung injury and clinical implications. J Appl Physiol (1985) 2021; 130: 877-891. 
3 Sayah W, Berkane I, Guermache I, et al. Interleukin-6, procalcitonin and neutrophil-to-lymphocyte ratio: potential immune-inflammatory parameters to identify severe and fatal forms of COVID-19. Cytokine 2021; 141: 155428.

4 Recovery Collaborative Group. Tocilizumab in patients admitted to hospital with COVID-19 (RECOVERY): a randomised, controlled, open-label, platform trial. Lancet 2021; 397: 1637-1645.

5 Gordon AC, Mouncey PR, Al-Beidh F, et al. Interleukin-6 receptor antagonists in critically ill patients with Covid-19. N Engl J Med 2021; 384: 1491-1502.

6 Tleyjeh IM, Kashour Z, Damlaj M, et al. Efficacy and safety of tocilizumab in COVID-19 patients: a living systematic review and meta-analysis. Clin Microbiol Infect 2021; 27: 215-227.

7 Voiriot G, Razazi K, Amsellem V, et al. Interleukin-6 displays lung anti-inflammatory properties and exerts protective hemodynamic effects in a double-hit murine acute lung injury. Respir Res 2017; 18: 64.

8 Pandolfi R, Barreira B, Moreno E, et al. Role of acid sphingomyelinase and IL- 6 as mediators of endotoxin-induced pulmonary vascular dysfunction. Thorax 2017; 72: 460-471.

9 Wang D, Fu B, Peng Z, et al. Tocilizumab in patients with moderate or severe COVID-19: a randomized, controlled, open-label, multicenter trial. Front Med 2021; 15: 486-494.

$10 \mathrm{Xu} \mathrm{X,} \mathrm{Han} \mathrm{M,} \mathrm{Li} \mathrm{T,} \mathrm{et} \mathrm{al.} \mathrm{Effective} \mathrm{treatment} \mathrm{of} \mathrm{severe} \mathrm{COVID-19} \mathrm{patients} \mathrm{with} \mathrm{tocilizumab.} \mathrm{Proc} \mathrm{Natl} \mathrm{Acad} \mathrm{Sci}$ USA 2020; 117: 10970-10975. 